\title{
Variabilité spatiale de la structure spécifique d'un peuplement ligneux et de l'endémicité en zone de montagne aux Comores
}

\author{
M.M. CHARAHABIL ${ }^{1,4 *}$, I. YAHAYA ${ }^{2}$, J-N. LABAT $^{3}$ et L.E. AKPO ${ }^{4}$ \\ ${ }^{1}$ Département d'agroforesterie, Université de Ziguinchor, B.P 523 Ziguinchor, Sénégal. \\ ${ }^{2}$ Centre National de Documentation et de Recherches Scientifiques des Comores, CNDRS, BP 169, \\ Moroni Comores. \\ ${ }^{3}$ UMR 7205 ; Origine Structure et Evolution de la Biodiversité, Département Systématique et Evolution 16 Rue \\ Buffon 75231 Paris, France. \\ ${ }^{4}$ Laboratoire d'Ecologie Végétale \& d'Eco-hydrologie (UCAD/FST) BP 5005 Dakar, Sénégal. \\ *Auteur correspondant, E-mail: mcharahabil@univ-zig.sn
}

\section{RESUME}

L'objet de ce travail est d'établir la variabilité de la structuration de la flore et de la végétation ligneuses de la forêt du Karthala. Des relevés de la végétation ont été réalisés dans le versant Ouest, dans versant Sud soumis à la mousson du Nord-Ouest et dans le versant Est soumis au Alizés du Sud-Est. La diversité (alpha et beta), l'organisation, le taux d'espèces endémiques, ont permis d'établir une nette variation dans la richesse floristique et la structure du peuplement végétal, ce qui témoigne une grande variabilité du patron habitat dans ces différents versants. Le versant Ouest est en effet, le légèrement plus diversifié (en moyenne 11,5 espèces au $100 \mathrm{~m}^{2}$ ) que le versant Est (11espèces). L'endémicité est plus élevée dans ces deux versants que le versant Sud. La densité du peuplement est par contre plus élevée dans le versant Sud, alors que la surface terrière est plus élevée à l'Est. L'étude du potentiel de régénération a montré que le peuplement régénère plus au Sud (55,5\% de jeunes plants) qu'à 1'Est $(24,2 \%)$ et à l'Ouest $(20,1 \%)$. L'étude de la beta diversité a montré une faible ressemblance floristique entre les versants.

(C) 2013 International Formulae Group. All rights reserved

Mots clés: Variabilité, végétation, indice de diversité alpha et beta, Karthala.

\section{INTRODUCTION}

Les Comores et l'ensemble des îles du Sud-Ouest de l'océan indien font parti des hotspots de la biodiversité mondiale (Labat et al., 2010). Malheureusement la structure de la végétation dans cet archipel et particulièrement du Karthala, la plus grande forêt climacique de la grande île est très peu étudiée. Une classification des différents types de formations forestières des Comores a été établie sur la base d'inventaires peu exhaustifs basés sur l'ethnobotanique et la pharmacopée traditionnelle (Adjanahoun et al., 1982). Ainsi la végétation des Comores comprendrait des formations naturelles (forêts denses et humides, formation arbustive ou buissonnante, la savane et la mangrove), des formations anthropisées, des plantations d'arbres (Eugenia aromtica, Artocarpus heterophilus, Mangifera indica...) et des zones des champs.

Ducatillon et Loup (1985) ont étudié l'arbre dans le paysage forestier des Comores et ont confirmé l'existence de ces formations 
en précisant la structure de la forêt qui est composée d'une forêt de basse altitude et d'une forêt humide de haute altitude au dessus de laquelle ont trouve une lande à éricacées tout près du sommet du volcan.

Des travaux récents (Keith et al., 2006), basés sur des inventaires faunistiques et floristiques dans l'archipel ont indiqué, qu'il existe deux types de végétation climaciques, à savoir les forêts caducifoliées et basses, de basses altitudes et les forêts denses humides de moyennes et hautes altitudes. Ces forêts entourent la montagne du Karthala dans ses trois versants : le versant Nord, le versant Sud et le versant Est. Ces situations renseignent que les différents versants du mont Karthala ne reçoivent pas les mêmes quantités de pluies annuelles du fait de leur exposition différentes aux vents qui arrivent dan l'île. Les versants Ouest et Sud sont, soumis directement aux vents du Nord Ouest (mousson) responsable des fortes précipitations. Le versant Est est soumis aux alizés du Sud Est, moins pluvieux.

$\mathrm{Au}$ niveau du mont Karthala, les forêts sont climaciques. Elles présentent avec celles de la Réunion des étages altimontains dans les îles du Sud Ouest de l'océan indien (Boullet, 2007).

La configuration et la topographie de la forêt du Karthala induisent une variation du patron habitat, cela entraîne une variation spatiale de la diversité et de la structure spécifique. La variation peut être d'origine naturelle ou préexistante (avant usages) ou accidentelle (après les usages) ou à une combinaison des deux effets (Trochon, 1996).

L'objectif de ce travail est d'établir de la structure spécifique du peuplement ligneux au niveau des trois versants du mont Karthala. Il s'agit de comparer la diversité floristique, l'endémicité, la structure verticale et le potentiel de régénération du peuplement dans les divers versants.

\section{MATERIEL ET METHODES Site d'étude}

L'étude a été réalisée dans la forêt du Karthala située dans la Grande Comores, l'île la plus jeune et la plus occidentale de l'archipel des Comores (Figure 1).

L'île est née entre 100 et 130000 ans, à l'issue d'un volcanisme océanique. Le massif du Karthala constitue son point culminant $(2361 \mathrm{~m})$ mais également celui de tout l'archipel. Il est découpé en 3 versants :

- un versant Ouest soumis aux vents du Nord Ouest (mousson africaine) apportant la saison des pluies, chaude et humide de novembre à avril ;

- un versant Sud, où se trouve la station la plus pluvieuse de l'île (station de Nioumbadjou) sous l'action du même vent ;

- et un versant Est soumis les alizés du SudEst responsable de la saison relativement sèche et fraiche de mai à octobre. On l'appelle communément versant sous le vent.

Les sols de la Grande-Comores sont très jeunes à l'image de l'île, ils sont perméables et peu altérés. Le climat est de type tropical humide sous l'influence de ces deux types de vents.

Les pluviométries moyennes annuelles varient entre $1398 \mathrm{~mm}$ et $5888 \mathrm{~mm}$. Les températures varient très peu de l'ordre de 4 ${ }^{\circ} \mathrm{C}$ avec une variation appréciable de l'humidité relative.

\section{Méthodes d'étude \\ Collecte des données}

Les relevés de la végétation ont été réalisés entre octobre 2008 et janvier 2009, et entre mai et juin 2010. Vue le degré de connaissance sur la flore des Comores, nous avons opté pour ces deux périodes différentes afin de maximiser les chances de récolter des spécimens fertiles, plus faciles à identifier pour l'instant. La technique d'échantillonnage par transect a été utilisée pour disposer les relevés. Dans chaque versant, la progression s'est effectuée le long des sentiers pédestres empruntés par les populations pour atteindre, suivant un gradient altitudinal, leur zone d'exploitation forestière et plus haut le sommet du Karthala. Au total 78 relevés 
M. M. CHARAHABIL et al. / Int. J. Biol. Chem. Sci. 7(3): 902-923, 2013

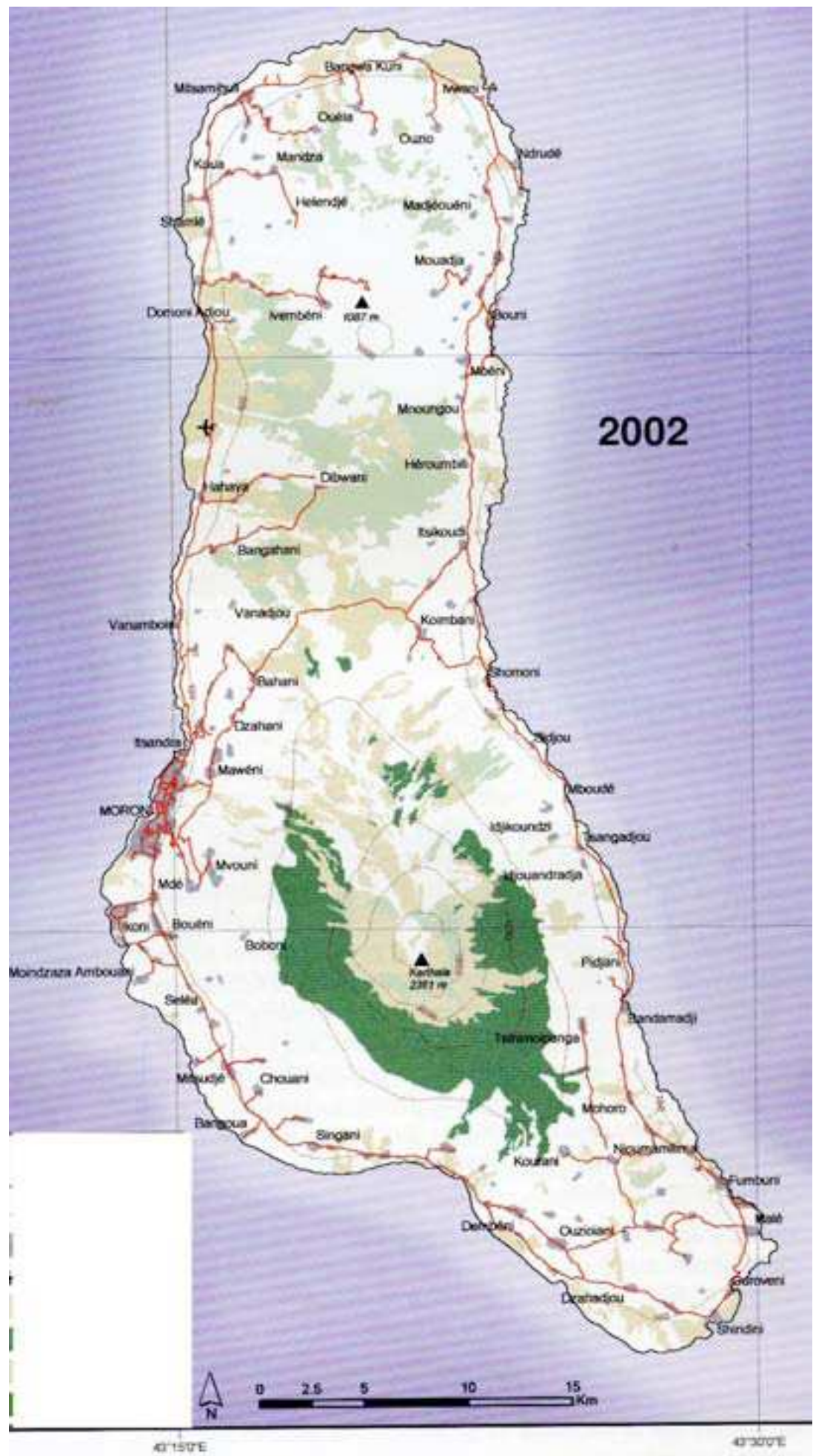

Figure 1: Localisation de la forêt du Karthala (couleur verte) dans la Grande Comores. 
ont été réalisés selon la répartition suivante :

- 32 dans le versant Ouest,

- 26 dans le versant Sud,

- et 20 dans le versant Est.

La surface de relevé est de $100 \mathrm{~m}^{2}$, elle équivaut à l'aire minimale de la zone définie par (Campbell et al., 1989). Au niveau de chaque relevé la liste floristique est établie en présence-absence et les paramètres structuraux quantitatifs de la végétation mesurés (densité, surface terrière, hauteur maximale des arbres, nombre de recrues). La dénomination des espèces a été réalisée sur place pour un certains nombre d'espèces et le reste identifié à l'herbier de Paris. La consultation de l'herbier des Comores et de la base Sonnerat a permis d'évaluer, pour chaque espèce rencontrée son niveau d'endémicité. La hauteur des arbres a été mesurée à l'aide d'une perche graduée.

\section{Traitement des données}

Les paramètres structuraux quantitatifs de la végétation ont été utilisés pour caractériser le peuplement ligneux globalement et par versant. Il s'agit :

- de la densité des arbres qui correspond au nombre d'individus par unité de surface

- de la surface terrière ou recouvrement basale qui désigne la surface occupé par les arbres au niveau du sol.

- de la régénération naturelle qui exprime le nombre de jeunes plants par unité de surface. On été considérés comme jeunes plants, les individus dont la circonférence à $10 \mathrm{~cm}$ du sol est inférieure à $10 \mathrm{~cm}$ (Charahabil et $a l$, 2008).

-de la stratification qui désigne la structure verticale du peuplement.

Pour comparer la structure et l'organisation du peuplement ligneux dans les trois versants, nous avons utilisé les indices de la diversité beta tel que :

- l'indice de similarité de Jaccard qui permet d'évaluer le degré de ressemblance floristique entre les versants.
$\mathrm{IS}=\mathrm{a} / \mathrm{a}+\mathrm{b}+\mathrm{c} \quad(\mathrm{b}:$ espèces propres $\mathrm{au}$ relevé $\mathrm{B}$; $\mathrm{c}$ : espèces propres au relevé $\mathrm{C}$; et $\mathrm{a}$ : espèces communes au relevé $\mathrm{B}$ et $\mathrm{C}$ ). Pour comparer le niveau d'organisation des groupements définis, nous avons utilisé l'indice de Shannon $\left(\mathrm{H}^{\prime}=-\sum \mathrm{Pi} \log \mathrm{Pi}\right.$; Pi étant la probabilité de rencontrer l'espèce de rang i) et l'indice d"'équitabilité de Piélou ou régularité $\left(\mathrm{R}=\mathrm{H}^{\prime} / \log 2 \mathrm{~S} ; \mathrm{H}^{\prime}\right.$ étant l'indice de Shannon et $\mathrm{S}$ la richesse spécifique). Nous avons choisi de combiner les deux indices du fait que les versants diffèrent entre eux tant par les effectifs des arbres que par la diversité floristique (Akpo et al., 1995).

La comparaison de la diversité et de la structure des versant a utilisé les indices de diversité et les paramètres structuraux quantitatives de la végétation telle que la densité, la surface terrière, la fréquence spécifique et la structure verticale des arbres et le potentiel de régénération des individus (nombre d'invendus dont le diamètre est inférieurs à $10 \mathrm{~cm}$ ).

\section{RESULTATS}

Caractéristiques générales du peuplement ligneux

\section{Composition}

La flore ligneuse inventoriée est riche de 95 espèces reparties entre 82 genres et 45 familles (Tableau 1). Avec 11 espèces reparties dans 7 genres, la famille des Rubiacées constitue la famille la plus importante de la zone. Elle est suivie des Euphorbiacées, 7 genres et 7 espèces et des Myrtacées 3 genres, 7 espèces. Le genre le plus important est le genre Psychotria composé de 4 espèces, suivi du genre Syzygium, 3 espèces.

\section{Fréquence spécifique}

L'étude de la répartition spatiale des espèces a montré que sur les 78 placeaux d'inventaires, les espèces les plus fréquentes sont :

Weinmannia comorensis $(57,7 \%)$, Ocotea comoriensis $(55,1 \%)$, Nuxia pseudodentata 
(47,4\%), Tambourissa comorensis $(46,1 \%)$ et Aphloia theaeformis (44,9\%). Ces 5 espèces sont présentes dans au moins la moitié des placeaux inventoriées. L'analyse des donnés n'a pas tenue compte du Psidium cattleyanum, qui est une espèce envahissante, présente pratiquement partout (Vos, 2004).

\section{Densité du peuplement}

Le peuplement ligneux est évalué à 1610 individus adultes $(\mathrm{dbh}>10 \mathrm{~cm})$. Les densités relatives ne sont pas très élevées. On distingue cependant, 3 espèces dont la densité relative est supérieure à $5 \%$. Il s'agit de Ocotea comoriensis (9,7\%), Tambourissa comorensis $(7,4 \%)$, et Weinmannia comorensis (7\%). Ces 3 espèces totalisent $24,2 \%$ de la végétation ligneuse.

La densité à l'hectare est évaluée à 2064 individus. Ocotea comoriensis compte 160 individus (7,75\%), Tambourissa comorensis $123(5,95 \%)$ et Weinmannia comorensis 116 individus $(5,62 \%)$.

\section{Surface terrière}

La surface terrière globale est évaluée à $164,74 \mathrm{~m}^{2} / \mathrm{ha}$. Elle est de $26,2 \mathrm{~m}^{2} /$ ha pour Weinmannia comorensis, $6,2 \mathrm{~m}^{2} / \mathrm{ha}$ pour Ocotea comoriensis et $2,9 \mathrm{~m}^{2} / \mathrm{ha}$ pour Tambourissa comorensis.

\section{Endémicité}

L'endémicité est évalué en taux d'espèces endémiques strictes à la Grande comore et en espèces endémiques à l'archipel des Comores. Ainsi Sur les 95 espèces ligneuses, nous avons recensé :

- 19 espèces endémiques à l'archipel des Comores, soit $20 \%$ de la flore ligneuse. Il s'agit de: Ophiocolea comorensis, Cassine sp, Erythroxylum nitidulum, Erythroxylum elegans, Croton humblotii, Ocotea comoriensis, Nuxia pseudodentata, Tambourissa comorensis, Myrica dentulata, Rapanea comorensis, Eugenia comorensis, Chassalia comorensis, Mapouria lavanchiei, Psychotria comorensis, Psychotria lavanchiei, Saldinia boiviniana, Gyrostipula comorensis, Vepris boiviniana, Vepris unifoliolata.
- 5 espèces endémiques à la Grande comore, soit $6,3 \%$ de la flore. Il s'agit de Ravenea hildebrandtii, Senecio humblotii, Philippia comoriensis, Scolopia coriacea, et Allophylus gardineri.

\section{Hauteur maximale des arbres.}

Le coefficient de variation qui est le rapport entre la moyenne et l'écartype est supérieur à 0,7 . Il indique une distribution hétérogène, donc instable au niveau du peuplement, ce qui signifie que les arbres ont une taille dispersée par rapport à la moyenne (13,1 m). La distribution des classes indique que les petits arbres $(5-10 \mathrm{~m})$ sont les plus dominantes, elles constituent $37,15 \%$ de l'effectif totale des ligneux. La strate arbustive constituée par la première classe est elle aussi très bien représentée $(25,75 \%)$. La canopée, formée par les très grands arbres dont la hauteur dépasse $40 \mathrm{~m}$ est par contre mal représentée elle ne réunit que $2,77 \%$ de l'effectif total.

L'examen de la structure des 3 espèces les plus représentées permet de mieux comprendre la structure globale.

Ocotea comoriensis (Figue 2B) présente la même distribution hétérogène presque identique à celle du peuplement (même coefficient de variation). Par contre, la hauteur moyenne est légèrement faible $(11,4 \mathrm{~m})$.

La courbe de hauteur de Tambourissa comorensis (Figure 2C) se présente sous la forme typique d'un $\mathrm{J}$ renversé. La strate arbustive (première classe), la plus dominante, renferme $35,4 \%$ de l'effectif totale des ligneux. Les écarts entres les 3 premières classes, qui réunissent $84,4 \%$ de l'effectif, sont plus ou moins régulières. C'est au niveau de la quatrième classe que l'on constate une rupture avec des effectifs qui deviennent très faibles. C'est une distribution typique d'une population équienne. La distribution est aussi hétérogène, avec un degré de dispersion moins faible $(\mathrm{Cv}=0,83)$. Par rapport à $O$. comoriensis. 
La distribution des par classes de hauteurs de Weinmania comorensis est assez homogène $(\mathrm{Cv}<0,7)$, ce la signifie que les hauteurs sont moins dispersées par rapport à la moyenne. On constate cependant, une faible représentation des effectifs dans les deux classes (30-35 m, 35-40 m) (Figure 2D) et l'on rencontre en revanche des grands arbres dont la hauteur dépasse $40 \mathrm{~m} \quad(10 \%$ de l'effectif total).

\section{Régénération du peuplement}

Nous avons recensé 2434 jeunes plants au niveau de la surface inventoriées. Ceci correspond à $48 \%$ de l'ensemble du peuplement ligneux. Dans cet ensemble de jeunes plants, Piper capense, et Eugenia aromatica sont les 2 espèces à forte régénération naturelle (Figure 2), avec respectivement $16 \%$ et $14 \%$ des jeunes plants. Les espèces dominantes présentent une faible régénération, elles renferment en tout $8,3 \%$ des jeunes plants.

Le peuplement est composé d'arbres de taille moyenne à grande, dominé par Ocotea comoriensis, Tambourissa comorensis et Weinmannia comorensis Ces espèces présentent un déficit de recrutement qui s'exprime par une faible présence de jeunes plants. De ces 3 espèces, c'est Weinnmania comorensis qui présente des individus de grande taille et c'est Tambourissa comorensis qui régénère mieux.

\section{Caractéristiques de la végétation suivant les versants}

\section{Espèces communes et espèces différentielles}

La liste de présence-absence des espèces dans les 3 versants (Tableau 1) résume l'information sur la variabilité de la diversité floristique. On distingue ainsi parmi les 95 espèces inventoriées 3 groupes d'espèces :

a) les espèces très communes, présentes dans les 3 versants, au nombre de 35 soit $36,8 \%$; b) les espèces peu communes, présentes dans 2 versants, au nombre de 21 soit 22,1\%. Dans ce groupe, on distingue des espèces communes entre :

- le versant Sud et le versant Est ; on rencontre 5 (Phoenix sp, Dicoryphe Thouarsii, Eugenia aromatica, Syzygium malaccense, et Mapouria lavanchiei.

-le versant Sud et le versant Ouest (6 espèces). Il s'agit de Croton humblotii, Cinnamomum zeylanicum, Leea guineensis, Lasiodiscus articulatus, Ravenea hildebrandtii, Citrus sinensis)

- le versant Est et le versant Ouest, Elles sont au total 10 espèces : Brachylaena ramiflora, Cycas thouarsii, Philippia comoriensis, Erythroxylum nitidulum, Acalypha filiformis, Apodytes dimidiata, Myrica dentulata, Morella spathulata, Rapanea comorensis et Chassalia comorensis.

c) Les espèces différentielles ou rencontrées au niveau d'un seul versant. Il s'agit de 39 espèces ainsi reparties:

- 11 pour le versant Sud (Senna septemtrionalis, Leucaena leucocepahala, Artocarpus communis, Piper umbellatum, Psychotria calothyris, Saldinia boiviniana, Vepris boiviniana, Filicium decipiens, Chrysophyllum boivinianum, Celtis philippensis, et Vitex beraviensis.

-11 pour le versant Est (Areca catechu, Senecio humblotii, Hubertia humblotii, Buxus moratii, Jatropha curcas, Khaya madagascariensis, Pisonia sechellarum, Pittosporum ochrosiifolium, Canthium bibracteatum, Gambeya boiviniana, et Pouzolzia guineensis

- et 17 pour le versant Ouest (Rhus natalensis, Polyscias felicis, Maytenus undata, Mystroxylon aethiopicum, Erythroxylum elegans, Wielandia leandriana, Scolopia coriacea, Woodfordia fruticosa, Urena lobata, Malleastrum sp, Oncostemum racemiferum, Syzygium cordatum, Psychotria lavanchiei, 
Psychotria sp, Tarenna grevei, Allophylus gardineri, et Boehmeria platyphylla.

\section{Similarité entre les versants}

Après avoir regroupé les espèces selon leur présence-absence, nous avons utilisé l'indice de similarité de Jaccard pour évaluer le degré de ressemblance entre la composition floristique des 3 versants.

Les valeurs de l'indice de similarité de Jacquard sont relativement faibles (Tableau 2). Il y a une faible ressemblance dans les cortèges floristiques des versants.

La valeur élevée de l'indice est trouvée entre l'Est et l'Ouest (26,3\% de similitude). Le versant Sud et le versant Ouest, bien que très proches représentent le plus faible indice de similitude $(17,6 \%)$.

\section{Paramètres de variabilité entre les sites}

La richesse spécifique moyenne (Tableau 3) qui exprime le nombre d'espèces inventoriées par relevé est plus élevée à l'Ouest; elle diminue légèrement à l'Est et puis au Sud.

La densité moyenne qui exprime le nombre d'individus en moyenne par relevé est plus élevée à l'Ouest $(22,9)$, elle diminue au Sud $(19,9)$ puis à l'Est $(18,1)$.

La densité à l'hectare est très élevée dans les 3 versants. Elle l'est plus à l'Ouest et au Sud.

Pour ce qui est de espèces dominantes on a constaté que :

Weinmannia comorensis est l'espèce la plus dominante dans le versant Sud. Elle totalise $6 \%$ de l'effectif totale des arbres. Ocotea comoriensis et Tambourissa comorrensis sont faiblement représentées dans ce versant. Elles sont devancées par d'autres espèces telles que Psychotria comorensis, les espèces du genre Eugenia, Macaranga boutonioides. Elles comptent respectivement $3,3 \%$ et $2,7 \%$ de l'effectif des arbres.
Dans le versant Est, Ocotea comoriensis et Tambourissa comorensis dominent. Elles ont respectivement comme densité relative $10,5 \%$ et $8,4 \%$. Weinmannia comorensis se positionne en troisième position avec $7,1 \%$.

A l'Ouest, c'est la même tendance: Ocotea comoriensis domine avec $9,6 \%$, suivie de Tambourissa comorensis 7\%, Weinmannia comorensis se positionne en quatrième position avec $4,6 \%$ derrière Apodites dimidiata $6 \%$.

La surface terrière qui exprime la surface occupée par les arbres à la surface du sol est plus élevée dans le versant Est, par rapport au Sud et à l'Ouest. Ce qui indique la présence de gros arbres dans ce versant. Weinmania comorensis avec une surface terrière de $116 \mathrm{~m}^{2} / \mathrm{ha}$ est l'espèce que présente les plus gros individus. Elle couvre à elle seule près de $50 \%$ de la surface terrière globale du versant. Ocotea comoriensis mesure 4,2 $\mathrm{m}^{2} / \mathrm{ha}$, et Tambourissa comorensis $3,5 \mathrm{~m}^{2} / \mathrm{ha}$.

Dans le versant sud, la surface terrière de Weinmania comorensis diminue considérablement, à l'image de la surface terrière globale, elle s'évalue à $20,6 \mathrm{~m}^{2} / \mathrm{ha}$ mais reste la plus élevée devant celle d' $O$. Comoriensis qui augment très légèrement $\left(4,8 \mathrm{~m}^{2} / \mathrm{ha}\right)$ et $T$. comorensis qui a légèrement diminué $\left(2,3 \mathrm{~m}^{2} / \mathrm{ha}\right)$. La valeur de la surface terrière de $W$. comorensis est encore plus faible à l'Ouest $\left(17,9 \mathrm{~m}^{2} / \mathrm{ha}\right), O$. comoriensis garde presque la même valeur $(4,2 \mathrm{~m} / \mathrm{ha})$ et T. comorensis retrouve la même valeur qu'à l'Est (3,5 $\left.\mathrm{m}^{2} / \mathrm{ha}\right)$.

Les indices de Shannon sont élevés (Is $>3,5$ ) dans les 3 versants; ce qui signifie que l'on est présence d'un peuplement bien diversifié, où les individus sont équitablement reparties entre les espèces (Ramade, 1984). Les indices de régularité qui témoigne une bonne organisation de la flore (Devineau et al., 1984). On ne note pas dans ces 
peuplements, des espèces à dominances manifestes.

Les espèces endémiques de large répartition sont plus abondantes à l'Ouest (14 espèces, soit $60,9 \%$ des espèces endémiques inventoriées). On en compte 12 au Sud soit $52,2 \%$ et 9 à l'Est soit $39,1 \%$ de la flore de ce versant. Pour ce qui est des 5 espèces endémiques strictes à la grande île, aucune espèce n'a été inventoriée au niveau du versant Sud. Les deux (2) espèces existent à l'Est (Philippia comoriensis, Senecio humblotii). Le versant Ouest est le plus riche en espèces endémiques strictes à la Grande comores ; sur les cinq espèces quatre (4) y ont été rencontrées (Ravenea hildebrandtii, Philippia comoriensis, Allophylus gardineri, Scolopia coriacea).

\section{Variabilité de la structure du peuplement}

Le coefficient de variation des trois courbes $(\mathrm{Cv}>5)$ indique une distribution hétérogène, donc instable (Figure 3 ).

Ces trois courbes présentent un pic commun qui correspond à la classe des arbres dont la hauteur est comprise entre 5 et $10 \mathrm{~m}$

On note cependant une variabilité spatiale. On remarque en effet, que la dispersion (hétérogénéité) est plus élevée dans le versant Sud $(\mathrm{cv}=0,83)$. Du point de vue de la répartition dans les différentes classes, on constate que le pic est aussi un peu plus élevé dans le versant Sud qu'à l'Est et à l'Ouest. On note aussi que la fréquence des arbustes (première classe) est plus élevée à l'Ouest par rapport au Sud et à l'Est. Au niveau de la loi de distribution de chaque courbe, on constate également que la courbe de distribution du versant Ouest est uni modale, avec un seul pic à ] 5-10] tandis que dans les deux autres versants, la distribution est bimodale avec 2 pics : un second pic à ] 35-40] pour le versant Est et à ] 40... pour le versant Sud. La Figure 4 représente la variabilité de la structure des 3 espèces dominantes.
La population de $T$. comorensis est constituée en majorité d'arbuste dans le versant Sud (Figure 5A). Dans le versant Ouest, les effectifs sont égaux dans les deux premières classes qui forment les 2 pics de la courbe. Au niveau du versant Est, la courbe de distribution est uni modale avec un seul pic au niveau de la seconde classe (] 5-10]).

La courbe de distribution d' $O$. Comoriensis (Figure 5B) est uni modale à l'Ouest avec un pic au niveau de la seconde classe (] 5-10]). Les arbustes ont les mêmes fréquences au Sud et à l'Est (20\%), ils sont un peu plus représentés à l'Ouest $(25 \%)$. Les grands arbres (hauteurs > $15 \mathrm{~m}$ ) sont par contre, inéquitablement reparties. Ils sont bien représentés au sud (33\%), un peu moins à l'Est $(17,5 \%)$, et à l'Ouest $(11,8 \%)$

La population de $W$. comorensis présente une distribution hétérogène, les hauteurs sont différentes d'un individu à un autre. On note de ce fait, 2 pics à l'Est à ] 510] et à ] 25-30] et au sud elle présente aussi 2 pics à ] 15-20] et à ] $40 .$. .

\section{Variation de la régénération selon les versants}

Globalement, la régénération est plus importante dans le versant Sud $(55,7 \%)$. Elle diminue à l'Est (24,2\%), et à l'Ouest $(20,1 \%)$. Les individus de ces espèces présentent un très faible potentiel de régénération naturelle et plus particulièrement Weinmannia comorensis (Figure 5). O. comoriensis et $T$. comorensis présentent quelques jeunes plants un peu partout.

La structure verticale de la végétation ligneuse (Figure 6) présente une distribution uni-modale suivant une loi polynomiale décroissante. 

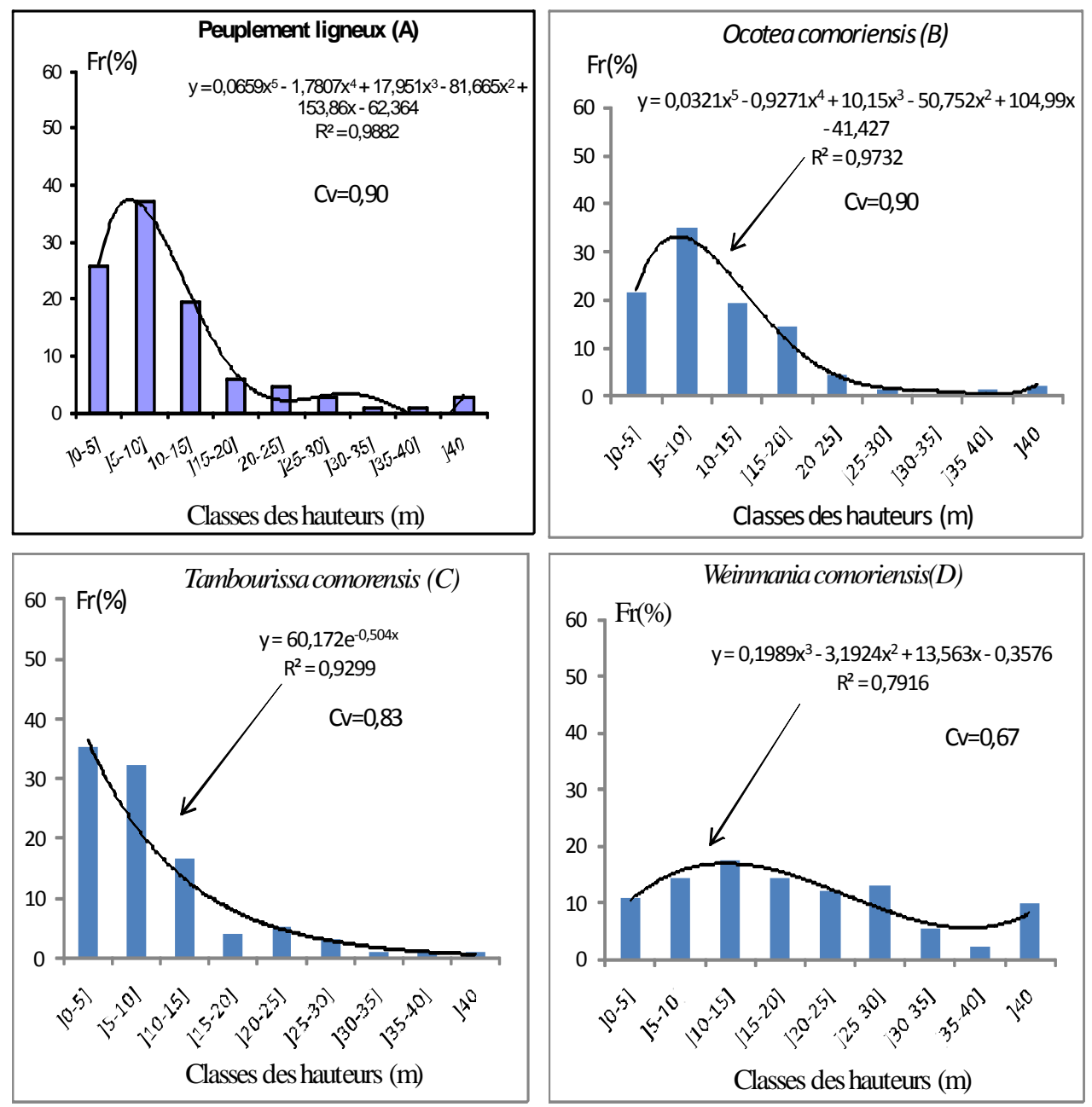

Figure 2: Structure verticale du peuplement ligneux(A), d'Ocotea comoriensis (B) Tambourissa comorensis (C), Weinmania comorensis (D).

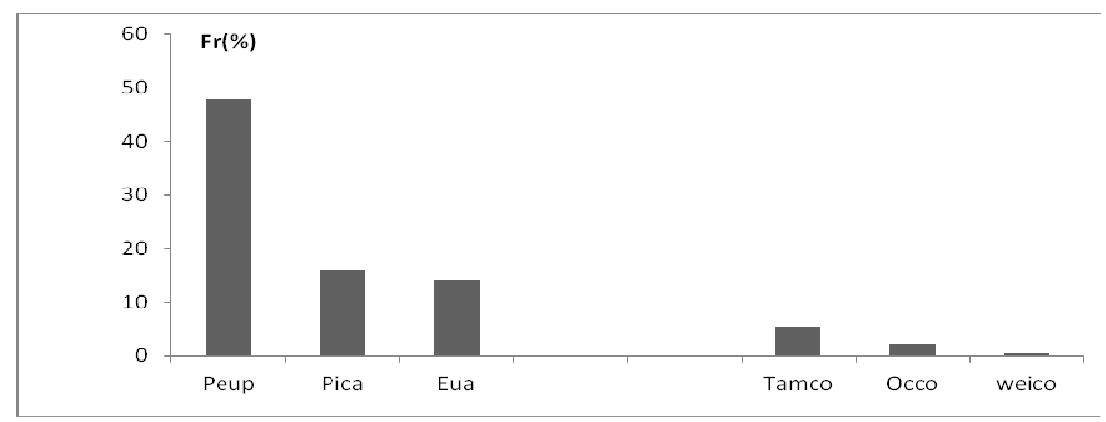

Figure 3 : Régénération de la végétation ligneuse. Peup = peuplement, Pica = Piper capens, Eua = Eugenia aromatica, Tamco $=$ Tambourissa comoriensis, Occo = Ocotea comoriensis, Weico $=$ Weinmannia comorensis. 


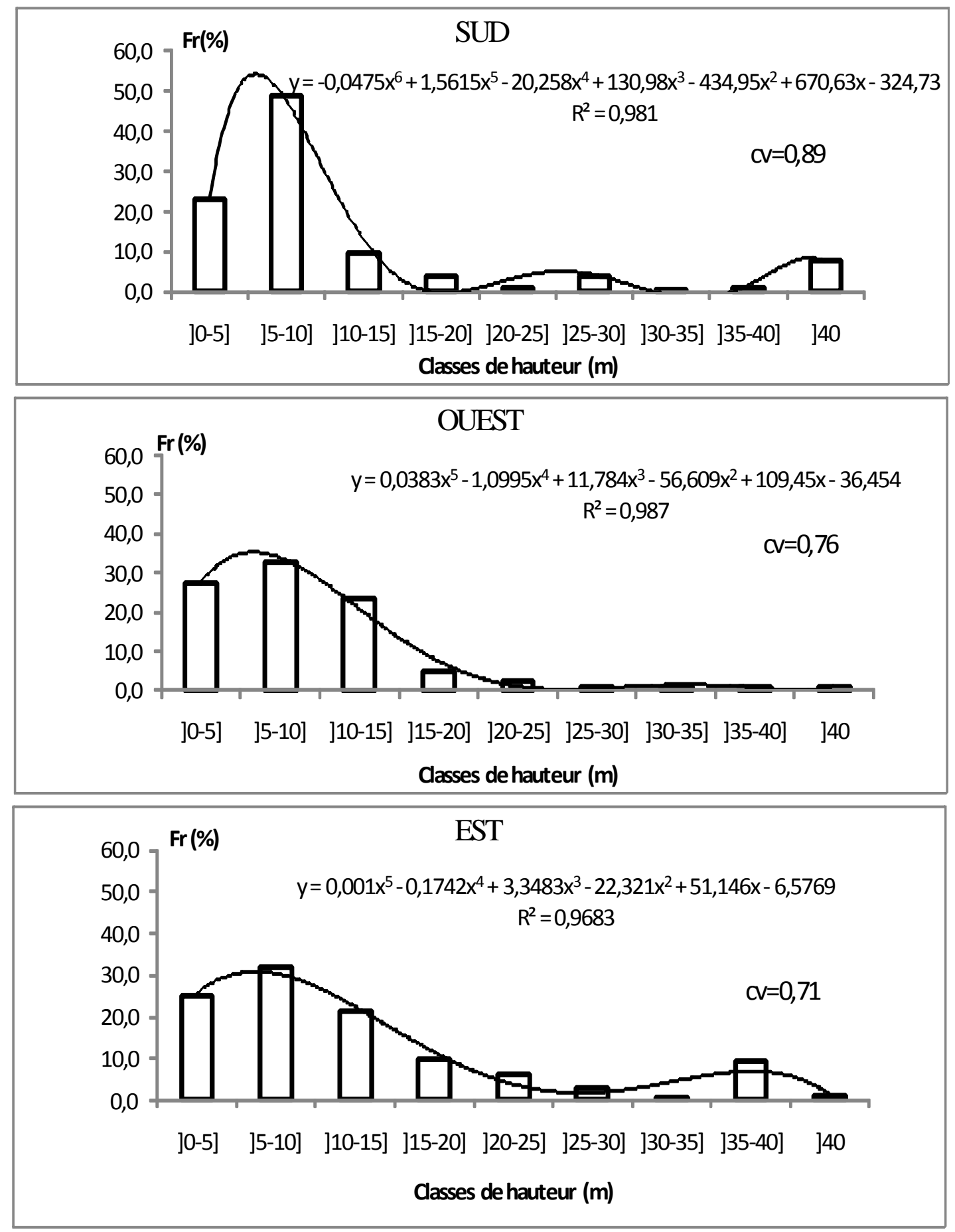

Figure 4 : Variabilité spatiale de la structure du peuplement. 


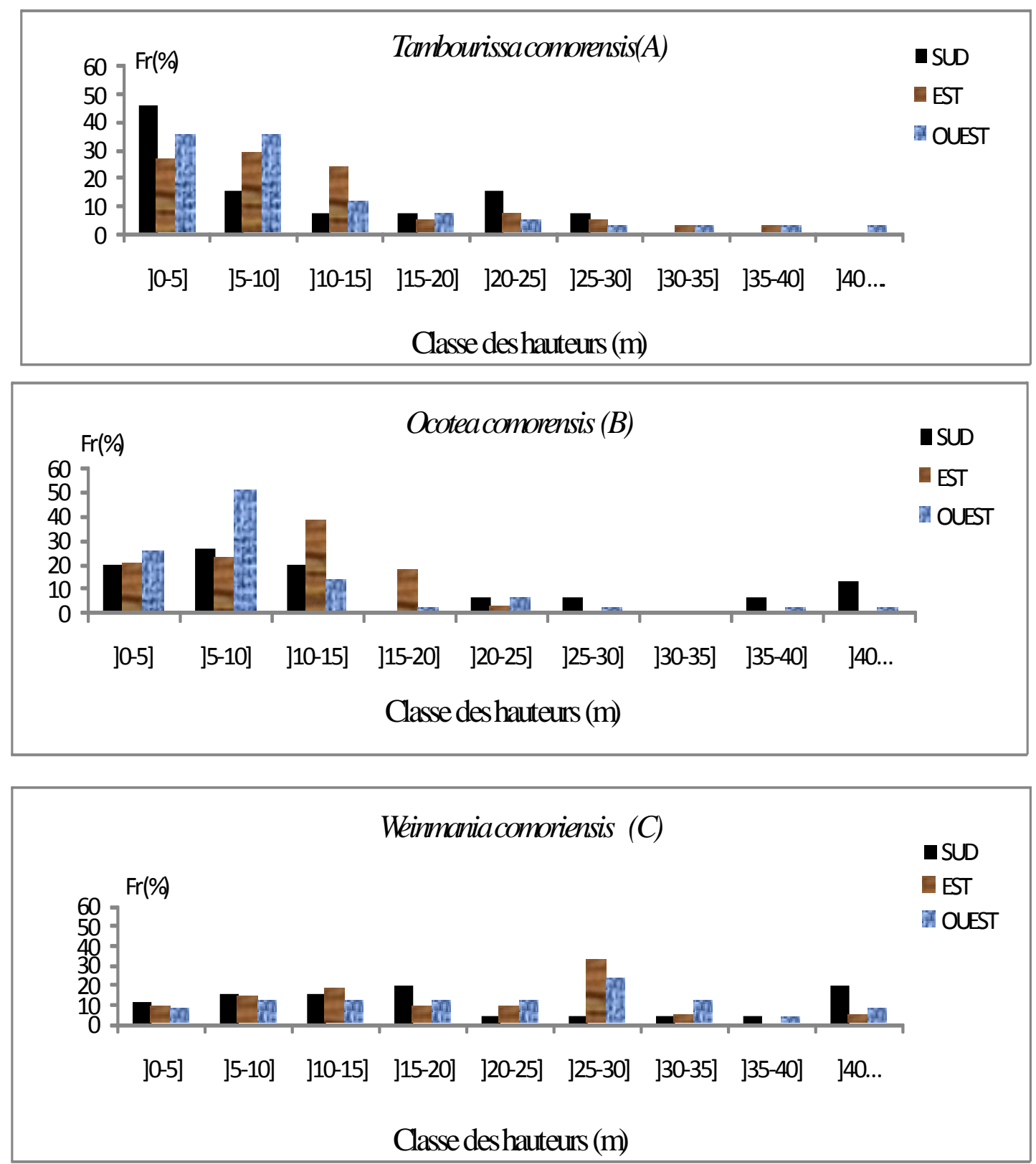

Figure 7 : Variabilité spatiale de la structure verticale des 3 espèces abondantes. 
M. M. CHARAHABIL et al. / Int. J. Biol. Chem. Sci. 7(3): 902-923, 2013

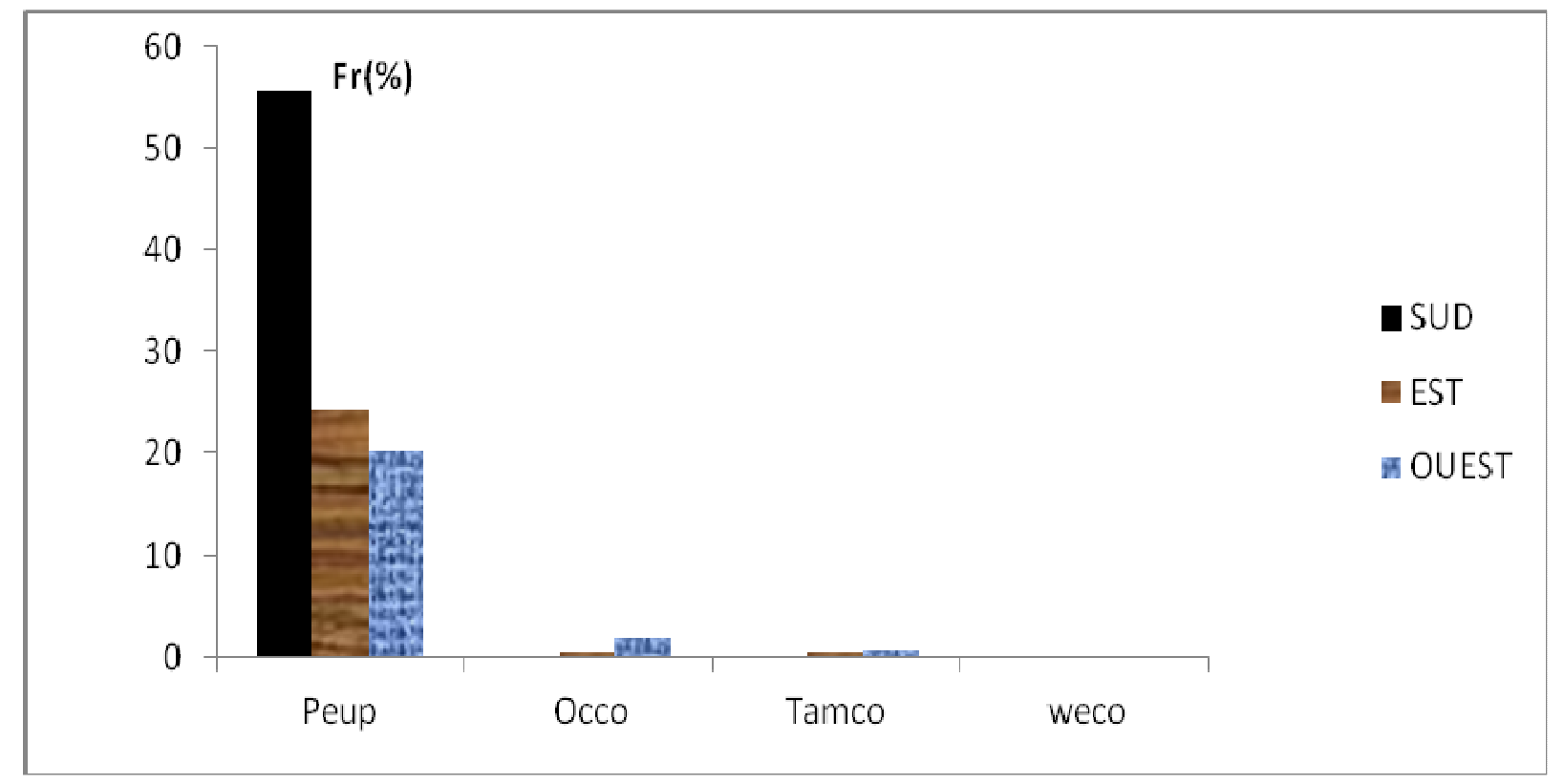

Figure 6 : Variation du potentiel de régénération suivant les versants.

Peup $=$ peuplement, Occo $=$ Ocotea comoriensis, tamco $=$ Tambourissa comorensis, weco $=$ Weinmania comorensis . 
Tableau 1: Matrice de présence- absence des espèces au niveau des versants.

\begin{tabular}{|c|c|c|c|c|c|}
\hline Familles & Genres & Espèces & Versant Sud & Versant Est & Versant Ouest \\
\hline & Mangifera & Mangifera indica $L$. & 1 & 1 & 1 \\
\hline Anacardiaceae & Rhus & Rhus natalensis Krauss & 0 & 0 & 1 \\
\hline Aphloiaceae & Aphloia & Aphloia theaeformis Benn. & 1 & 1 & 1 \\
\hline \multirow{4}{*}{ Araliaceae } & Schefflera & Schefflera myriantha (Baker) Drake & 1 & 1 & 1 \\
\hline & Polyscias & Polyscias felicis Bernardi & 0 & 0 & 1 \\
\hline & Gastonia & Gastonia duplicata Thouars ex Baill. & 1 & 1 & 1 \\
\hline & Cussonia & Cussonia spicata Thunb. & 1 & 1 & 1 \\
\hline \multirow{4}{*}{ Arecaceae } & Cocos & Cocos nucifera $L$ & 1 & 1 & 1 \\
\hline & Ravenea & Ravenea hildebrandtii C.D. Bouché & 1 & 0 & 1 \\
\hline & Areca & Areca catechu $L$ & 0 & 1 & 0 \\
\hline & Phoenix & Phoenix sp L. & 1 & 1 & 0 \\
\hline \multirow{3}{*}{ Asteraceae } & Brachylaena & Brachylaena ramiflora (D.C) humb.var comoriensis humb & 0 & 1 & 1 \\
\hline & Senecio & Senecio humblotii Klatt & 0 & 1 & 0 \\
\hline & Hubertia & Hubertia humblotii (Klatt) C.Jeffrey & 0 & 1 & 0 \\
\hline Begoniaceae & Begonia & Begonia comorensis A. DC. ex Warb. & 1 & 1 & 1 \\
\hline Bignoniaceae & Ophiocolea & Ophiocolea comorensis H. Perrier & 1 & 1 & 1 \\
\hline Buxaceae & Buxus & Buxus moratii G.E. Schatz \& Lowry & 0 & 1 & 0 \\
\hline \multirow{3}{*}{ Celastraceae } & Cassine & Cassine sp L. & 1 & 1 & 1 \\
\hline & Maytenus & Maytenus undata (Thumb.) Blakelock & 0 & 0 & 1 \\
\hline & Mystroxylon & Mystroxylon aethiopicum (Thunb.) Loes. & 0 & 0 & 1 \\
\hline Cunoniaceae & Weinmannia & Weinmannia comorensis Tul. & 1 & 1 & 1 \\
\hline
\end{tabular}


M. M. CHARAHABIL et al. / Int. J. Biol. Chem. Sci. 7(3): 902-923, 2013

\begin{tabular}{|c|c|c|c|c|c|}
\hline Cycadaceae & Cycas & Cycas thouarsii Gaudich. & 0 & 1 & 1 \\
\hline Ebenaceae & Euclea & Euclea mayottensis H. Perrier & 1 & 1 & 1 \\
\hline Ericaceae & Philippia & Philippia comoriensis Engl & 0 & 1 & 1 \\
\hline \multirow[b]{2}{*}{ Erythroxylaceae } & \multirow[b]{2}{*}{ Erythroxylum } & Erythroxylum nitidulum Baker & 0 & 1 & 1 \\
\hline & & Erythroxylum elegans Baill. & 0 & 0 & 1 \\
\hline \multirow{7}{*}{ Euphorbiaceae } & Fluggea & Fluggea virosa Baill. & 1 & 1 & 1 \\
\hline & Phyllanthus & Phyllanthus niruri $L$. & 1 & 1 & 1 \\
\hline & Macaranga & Macaranga boutonioides Baill. & 1 & 1 & 1 \\
\hline & Jatropha & Jatropha curcas $L$. & 0 & 1 & 0 \\
\hline & Acalypha & Acalypha filiformis Poir. & 0 & 1 & 1 \\
\hline & Croton & Croton humblotii Baill. & 1 & 0 & 1 \\
\hline & Wielandia & $\begin{array}{c}\text { Wielandia leandriana (Petra Hoffm. \& McPherson) Petra Hoffm. \& } \\
\text { McPherson }\end{array}$ & 0 & 0 & 1 \\
\hline \multirow[t]{2}{*}{ Fabaceae } & Senna & Senna septemtrionalis (viv.) H.S.Irwin & 1 & 0 & 0 \\
\hline & Albizzia & Albizzia glabereima Benth & 1 & 1 & 1 \\
\hline Flacourtiaceae & Scolopia & Scolopia coriacea Tul. & 0 & 0 & 1 \\
\hline Hamamelidaceae & Dicoryphe & Dicoryphe Thouars & 1 & 1 & 0 \\
\hline \multirow[t]{2}{*}{ Icacinaceae } & Apodytes & Apodytes dimidiata E. Mey. ex Arn. & 0 & 1 & 1 \\
\hline & Ocotea & Ocotea comoriensis Kosterm. & 1 & 1 & 1 \\
\hline Lauraceae & Cinnamomum & Cinnamomum zeylanicum Breyn. & 1 & 0 & 1 \\
\hline \multirow[t]{2}{*}{ Leaceae } & Leea & Leea guineensis G.Don f. comoriensis Desc. & 1 & 0 & 1 \\
\hline & Nuxia & Nuxia pseudodentata Gilg & 1 & 1 & 1 \\
\hline \multirow[t]{2}{*}{ Loganiaceae } & Anthocleista & Anthocleista grandiflora Gilg & 1 & 1 & 1 \\
\hline & Buddleja & Buddleja indica Lam. & 1 & 1 & 1 \\
\hline Lyrthaceae & Woodfordia & Woodfordia fruticosa (L.) Kurz & 0 & 0 & 1 \\
\hline Malvaceae & Urena & Urena lobata $L$. & 0 & 0 & 1 \\
\hline
\end{tabular}


M. M. CHARAHABIL et al. / Int. J. Biol. Chem. Sci. 7(3): 902-923, 2013

\begin{tabular}{|c|c|c|c|c|c|}
\hline \multirow[t]{2}{*}{ Meliaceae } & Khaya & Khaya madagascariensis Jum. \& Perr. & 0 & 1 & 0 \\
\hline & Malleastrum & Malleastrum J.-F. Leroy & 0 & 0 & 1 \\
\hline Mimosaceae & Leucaena & Leucaena leucocepahala (Lam) de Wit & 1 & 0 & 0 \\
\hline Monimiaceae & Tambourissa & Tambourissa comorensis Lorence & 1 & 1 & 1 \\
\hline \multirow{4}{*}{ Moraceae } & \multirow[t]{2}{*}{ Ficus } & Ficus pyrifolia Burm. $f$. & 1 & 1 & 1 \\
\hline & & Ficus sp L. & 1 & 1 & 1 \\
\hline & \multirow[t]{2}{*}{ Artocarpus } & Artocarpus communis J.R. Forst. \& G. Forst. & 1 & 0 & 0 \\
\hline & & Artocarpus heterophyllus & 1 & 1 & 1 \\
\hline \multirow[b]{2}{*}{ Myricaceae } & Myrica & Myrica dentulata Baill. & 0 & 1 & 1 \\
\hline & Morella & Morella spathulata (Mirb.) Verdc. \& Polhill & 0 & 1 & 1 \\
\hline \multirow[t]{2}{*}{ Myrsinaceae } & Rapanea & Rapanea comorensis Mez & 0 & 1 & 1 \\
\hline & Oncostemum & Oncostemum racemiferum $\mathrm{Mez}$ & 0 & 0 & 1 \\
\hline \multirow{7}{*}{ Myrtaceae } & Psidium & Psidium cattleyanum var. coriaceum (Mart. ex O. Berg) Kiaersk. & 1 & 1 & 1 \\
\hline & \multirow{3}{*}{ Eugenia } & Eugenia aromatica (L.) Baill. & 1 & 1 & 0 \\
\hline & & Eugenia sp $L$ & 1 & 1 & 1 \\
\hline & & Eugenia comorensis H. Perrier & 1 & 1 & 1 \\
\hline & \multirow{3}{*}{ Syzygium } & Syzygium malaccense (L.) Merr. \& L.M. Perry & 1 & 1 & 0 \\
\hline & & Syzygium jambos (L.) Alston & 1 & 1 & 1 \\
\hline & & Syzygium cordatum (Hochst.) & 0 & 0 & 1 \\
\hline Nyctaginaceae & Pisonia & Pisonia sechellarum F.Friedmann & 0 & 1 & 0 \\
\hline \multirow[t]{2}{*}{ Piperaceae } & \multirow[t]{2}{*}{ Piper } & Piper capense L. $f$. & 1 & 1 & 1 \\
\hline & & Piper umbellatum L. & 1 & 0 & 0 \\
\hline Pittosporaceae & Pittosporum & Pittosporum ochrosiifolium Bojer & 0 & 1 & 0 \\
\hline \multirow[t]{3}{*}{ Rhamnaceae } & Lasiodiscus & Lasiodiscus articulatus Capuron & 1 & 0 & 1 \\
\hline & \multirow[t]{2}{*}{ Canthium } & Canthium sp. Lam & 1 & 1 & 1 \\
\hline & & Canthium bibracteatum (Backer) Hiern & 0 & 1 & 0 \\
\hline
\end{tabular}


M. M. CHARAHABIL et al. /Int. J. Biol. Chem. Sci. 7(3): 902-923, 2013

\begin{tabular}{|c|c|c|c|c|c|}
\hline \multirow{9}{*}{ Rubiaceae } & Chassalia & Chassalia comorensis Bremek. & 0 & 1 & 1 \\
\hline & Mapouria & Mapouria lavanchiei Bremek. & 1 & 1 & 0 \\
\hline & \multirow{4}{*}{ Psychotria } & Psychotria comorensis Bremek. & 1 & 1 & 1 \\
\hline & & Psychotria calothyris (Bremek.) A.P.Davis \& Govaerts & 1 & 0 & 0 \\
\hline & & Psychotria lavanchiei Bremek. & 0 & 0 & 1 \\
\hline & & Psychotria sp L. & 0 & 0 & 1 \\
\hline & Saldinia & Saldinia boiviniana (Baill.) Bremek. & 1 & 0 & 0 \\
\hline & Tarenna & Tarenna grevei (Drake) Homolle & 0 & 0 & 1 \\
\hline & Gyrostipula & Gyrostipula comorensis Leroy & 1 & 1 & 1 \\
\hline \multirow[t]{3}{*}{ Rutaceae } & Citrus & Citrus sinensis (L.) Osbeck & 1 & 0 & 1 \\
\hline & Vepris & Vepris boiviniana (Baill.) Mziray & 1 & 0 & 0 \\
\hline & & Vepris unifoliolata Baill. & 1 & 1 & 1 \\
\hline \multirow[t]{2}{*}{ Sapindaceae } & Allophylus & Allophylus gardineri Summerh. & 0 & 0 & 1 \\
\hline & Filicium & Filicium decipiens (Wight \& Arn.) Thwaites & 1 & 0 & 0 \\
\hline \multirow[t]{2}{*}{ Sapotaceae } & Chrysophyllum & Chrysophyllum boivinianum (Pierre) Baehn & 1 & 0 & 0 \\
\hline & Gambeya & Gambeya boiviniana Pierre & 0 & 1 & 0 \\
\hline Solanaceae & Solanum & Solanum mauritianum Scop & 1 & 1 & 1 \\
\hline Sterculiaceae & Dombeya & Dombeya sp. Cav & 1 & 1 & 1 \\
\hline Ulmaceae & Celtis & Celtis philippensis Blanco & 1 & 0 & 0 \\
\hline \multirow{2}{*}{ Urticaceae } & Pouzolzia & Pouzolzia guineensis Benth. & 0 & 1 & 0 \\
\hline & Boehmeria & Boehmeria platyphylla D.Don. var. macrostachya (Wight) Wedd. & 0 & 0 & 1 \\
\hline \multirow[t]{2}{*}{ Verbenaceae } & Vitex & Vitex doniana Sweet & 1 & 1 & 1 \\
\hline & & Vitex beraviensis Vatke & 1 & 0 & 0 \\
\hline
\end{tabular}


Tableau 2: Indice de similarité de Jacquard entre les 3 versants.

\begin{tabular}{lccc}
\hline Indice de Jacquard $(\%)$ & Versant sud & Versant Est & Versant Ouest \\
\hline Versant sud & 1 & 18,5 & 17,6 \\
Versant Est & 18,5 & 1 & 26,3 \\
Versant Ouest & 17,6 & 26,3 & 1 \\
\hline
\end{tabular}

Tableau 3 : Paramètres de variabilité de la structure de la végétation ligneuse.

\begin{tabular}{lccc}
\hline Paramètres & Versant sud & Versant est & Versant ouest \\
\hline Richesse spécifique totale & 57 & 61 & 67 \\
Richesse spécifique moyenne & 9,9 & 11 & 11,4 \\
Densité moyenne & 19,9 & 18,1 & 22,9 \\
Densité à l'hectare & 1999,3 & 1814,2 & 1001,4 \\
Surface terrière (m²/ha) & 188,4 & 236,6 & 93,03 \\
Indice de Shannon & 4,4 & 4,9 & 4,7 \\
Indice de régularité & 0,77 & 0,77 & 0,80 \\
Endémisme large & 12 & 9 & 14 \\
Endémisme stricte & 1 & 3 & 4 \\
\hline
\end{tabular}

\section{DISCUSSION}

Nous avons établi la variabilité spatiale de la flore et de la structure de la végétation du Karthala. Au total 95 espèces ligneuses reparties entre 82 genres et 45 familles ont été inventoriées.

La famille des rubiacées constitue la famille la plus dominante. Les récents inventaires (Keith et al., 2006) avaient classé cette famille en quatrième position derrière les Ptéridophytes, les Fabacées, et les orchidées. $\mathrm{Du}$ faite que l'essentiel de la liste publiée, extrait de la base Sonnerat (www.sonnerat.org), provenait d'inventaires réalisés par des Ptéridologues et par un spécialiste des légumineuses (Labat et Pascal, 1999 ; Labat et Du Puy, 1997). De plus, les orchidées, constituent une très grande famille notamment dans le sous bois des forêts humides et ont beaucoup intéressé ces botanistes du fait de leur endémicité (Pascal, $1997 ; 2002)$.
La densité et la surface terrière sont élevées. (2064 individus/ha et 164,74 m²/ha), ce sont des valeurs typiques des forêts tropicales humides (Holl, 2002). La flore est légèrement dominée en terme de fréquence et de densité par Ocotea comoriensis, Tambourissa comorensis et Weinmannia comorensis.

W. Comorensis est plus fréquente par rapport aux deux autres espèces, elle présente aussi la surface terrière la plus élevée. Par contre la densité est plus élevée chez $O$. comoriensis et $T$. comorensis.

Les espèces endémiques des Comores représentent (20\%) de la flore ligneuse inventoriée. C'est un pourcentage voisin de celui de Sao Tomé, mais très en dessous de celui d'autres îles océaniques telles que Galápagos (41\%), Canaries (53\%) et Nouvelles Calédonie (79\%), Pascal et al., (2001). Ce pourcentage est plus élevé que celui indiqué par Keith et al. (2006), qui 
évalue le taux des espèces endémiques de l'archipel des Comores à 14,46\%. Par contre les espèces endémiques de la Grande île des Comores $(6,3 \%)$, constitue un taux très en dessous de la valeur estimée par les mêmes auteurs $(11,55 \%)$. C'est un pourcentage proche de celui évalué à Mayotte (7\%). Les différences s'établissent du faite que nos inventaires ne concernant que les ligneux inventoriées dans la forêt du Karthala alors que les taux estimés par nos prédécesseurs concernent toutes les types biologiques et dans tous les habitats du pays.

La hauteur moyenne des arbres est estimée à $13 \mathrm{~m}$, la quasi-totalité des individus est concentrée dans les premières classes, qui réunissent les arbustes et les arbres à hauteur moyenne. Les très grands arbres qui survolent la canopée représentent moins de $3 \%$ des effectifs totales. Ce qui confirme les observations de (Ducathillon et Loup, 1985). Parmi les 3 espèces les plus fréquentes c'est W. comorensis qui présente des grands arbres. Près de $50 \%$ de ses individus sont hauts de plus de $20 \mathrm{~m}$. T. comorensis et $O$. comoriensis sont constitués majoritairement par des arbustes et des petits arbres. Cette stratification est liée à la nature même des espèces mais aussi à l'exploitation pour le bois. Les grands arbres sont le plus souvent coupés et utilisées comme bois dans la construction des maisons et des pirogues.

Le peuplement ligneux régénère bien, les jeunes plants représentent $48 \%$ des effectifs totales. Ces résultats se rapprochent de ceux de Pascal (2002). Ce qui signifie que sous un arbre on retrouve en moyenne deux rejets. Les conditions climatiques et environnementales du Karthala sont donc favorables au rajeunissement perpétuel du couvert végétal. Rajemisson (2010) a trouvé des résultats similaires dans les hautes terres $\mathrm{du}$ centre de Madagascar. Les espèces qui régénèrent le plus sont :
- Piper capense (Piperaceae) une espèce que l'on trouve dans la forêt humide des hautes altitudes dont les graines sont utilisées en médecines traditionnelles contre les maux de dents (Zainaba, 2009). La multiplication de cette espèce serait donc végétative. L'accessibilité difficile des hautes altitudes protège aussi l'espèce de l'usage abusive et lui permet dans les conditions qui lui sont très favorables de se multiplier considérablement.

- Eugenia aromatica (Myrtaceae), le giroflier c'est une plante aromatique et médicinale. On le rencontre dans les zones des cultures dans les basses altitudes et très rarement dans la forêt. C'est une culture de rente très active aux Comores. Les Comores constituent le cinquième producteur mondial de clou de girofle avec une production annuelle de 124392 tonnes en 2004. Malgré le fait que ce sont les organes reproducteurs (fleurs) qui font l'objet de l'exploitation, cette espèce présente un potentiel de régénération exceptionnel.

Les espèces les plus fréquentes présentent un faible taux de régénération, notamment $W$. comoriensis qui se présente en peuplement vieillissant. Le bois de cette espèce est fortement prisé par les populations pour sa qualité (Ducatillon et Loup, 1985), ce qui entraine un déficit crucial de jeunes plants. Parmi ces 3 espèces, c'est T. comorensis présente le taux de régénération le plus élevé $(5 \%)$. O. comoriensis régénère mal $(2 \%$ de jeunes plants). L'explication que l'on peut tirer de cette structure du peuplement ligneux est que le paysage forestier est actuellement dominé par des espèces vieillissant, sous l'effet de l'action anthropique et que le renouvellement se fera par d'autres espèces moins utilisées par la population ou sinon par des espèces à croissance rapide comme le Psidium cattleyanum si l'on ne contrôle pas sa dynamique d'invasion.

Les variations spatiales du peuplement ligneux permettent de particulariser chaque 
versant. Il en est ressorti que $41,5 \%$ des ligneux inventoriés se situent dans le versant sud contre $37,7 \%$ à l'Est et 20,8\% à l'Ouest. La richesse floristique est par contre plus élevée à l'Ouest. En effet, 67 espèces ont été inventoriées pour en moyenne 11,5 espèces par unité d'inventaire. La diversité est aussi plus élevée à l'Est (11 espèces par relevé) par rapport à l'Est (10 espèces par relevé). Cette asymétrie de la structure spécifique résulte de la réponse des espèces vis-à-vis de l'action du vent, de la topographie mais aussi de l'action de l'homme (Sarrasin, 2006).

$W$. comorensis domine dans le versant Sud alors que $O$. comorensis et $T$. comorensis sont codominantes dans l'Est et l'Ouest. Cette variabilité dans la prédominance des espèces serait liée aux mécanismes d'adaptation des espèces. W. comoriensis serait plus adaptée au sud et les deux autres espèces l'Ouest et l'Est.

La surface terrière est aussi variable suivant les versants. Elle est plus élevée à l'Est $\left(236,6 \mathrm{~m}^{2} / \mathrm{ha}\right)$ avec $W$. comorensis qui présente près de $50 \%$ de cette surface terrière. Elle est évaluée à $188,4 \mathrm{~m}^{2} / \mathrm{ha}$ au Sud, elle devient faible à l'Ouest $\left(93,03 \mathrm{~m}^{2} / \mathrm{ha}\right)$. Ce résultat traduit que l'on trouverait plus de gros arbres en particulier W. comorensis (Mrikoudi en langue locale) à l'Est. Nos observations ont montré en effet que c'est dans des localités comme « Kandzilé », « kourani », «Nioumamili $m a »$, toutes situé à l'Est, que l'on sort des grosses planches utilisées dans l'artisanat, la menuiserie et la construction des pirogues (Photo 1).

L'étude de la stratification verticale, a indiqué que le peuplement présente une distribution hétérogène (instable). Il existe une grande dispersion de la taille des individus par rapport à la valeur moyenne. Le peuplement est globalement dominé par des arbres dont la hauteur est située entre 5 et $10 \mathrm{~m}$. Il existe cependant une variabilité de cette organisation suivant le versant. D'abord, cette classe d'individus traduisant les arbres de hauteur moyenne est plus importante au Sud que dans les autres versants. Les arbustes qui constituent la première classe de l'analyse (entre 0 et $5 \mathrm{~m}$ ) sont par contre plus importants à l'Ouest. Les arbres hauts de plus de $20 \mathrm{~m}$ sont rares, on retrouve des grands arbres au niveau du versant Sud mais surtout à l'Est où ils peuvent dépasser $35 \mathrm{~m}$ de hauteur. Certaines classes de hauteur sont très faiblement représentées, pour ne pas dire inexistant (30 à $35 \mathrm{~m}$ ). Ce déficit se traduirait par une préférence d'exploitation des bois atteignant cette hauteur.

Parmi les trois espèces dominantes, c'est W. comorensis qui présente une distribution homogène, ce qui signifie que les écarts entre la taille des individus sont faibles. L'exploitation de l'arbre, se fait lorsqu'il atteint une taille bien définit, elle correspond à la classe la plus faiblement représentée. Les gros arbres de l'espèce sont plutôt présents au Sud.

Les deux autres espèces présentent une distribution hétérogène, traduisant des écartypes trop grandes. La majorité des individus est concentrés dans les classes inférieures (arbustes et les arbres de hauteur moyens). Ce sont des populations équiennes bien équilibrées. Entre ces deux espèces, les arbustes sont plus nombreux chez $T$. comorensis et les arbres de hauteur moyenne chez $O$. comoriensis, qui présente aussi plus de grands arbres notamment dans le versant Sud. 


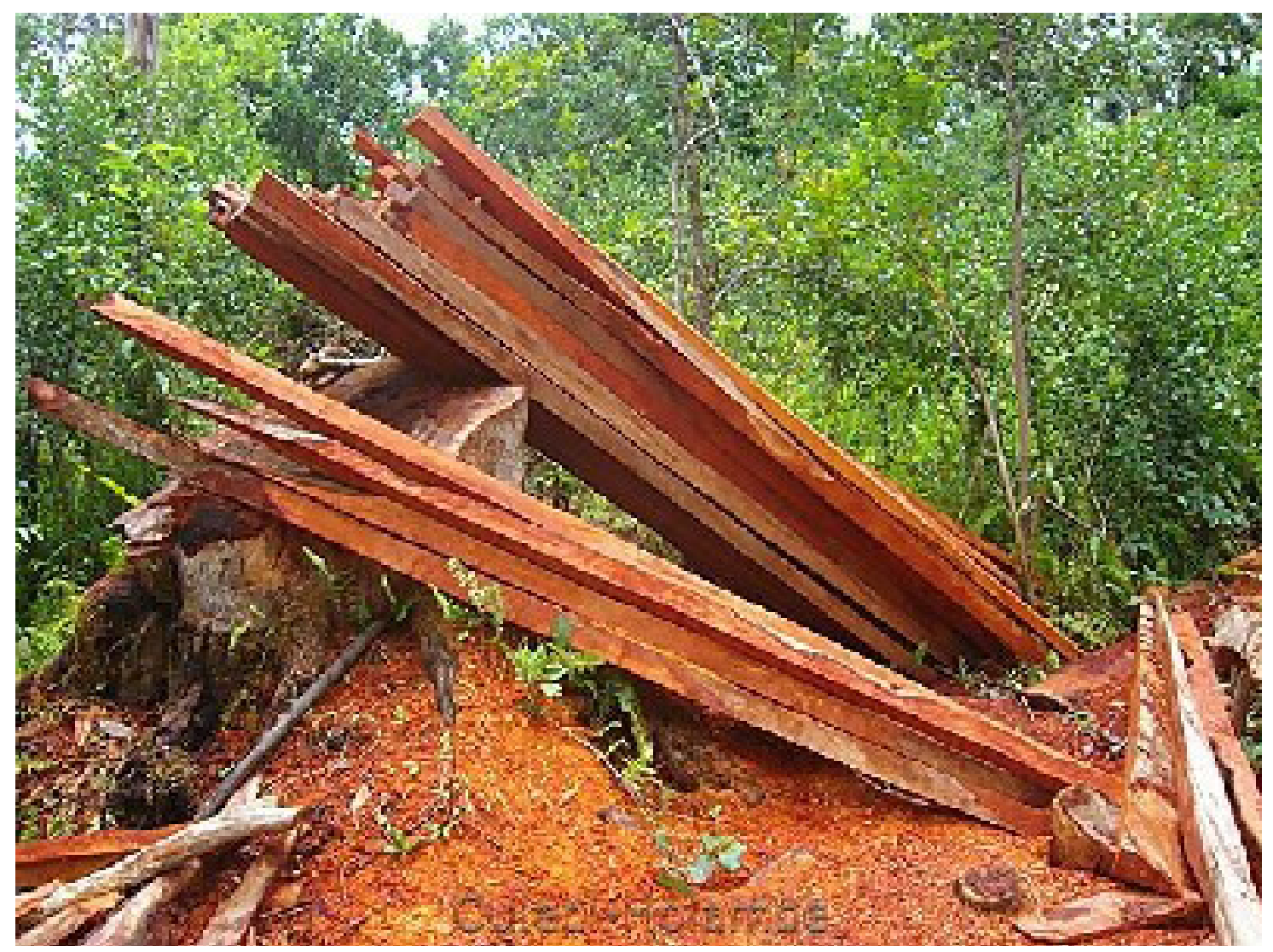

Photo 1 : Exploitation du bois de Weinnmania comoriensis dans la forêt (Photo Ramadane).

Le peuplement ligneux régénère bien dans le versant Sud. Il l'est moins à l'Est et à l'Ouest. Les espèces les plus fréquentes régénèrent très mal notamment $W$. comorensis.

Dans le versant ouest, O. comoriensis présente $1 \%$ de jeunes plants et $T$. comorensis un peu moins. Les jeunes plants inventoriés appartiennent à d'autres espèces moins fréquentes qui tendent à assurer le renouvèlement de la couverture végétale.

\section{Conclusion}

Ce travail a permis de mettre en évidence les particularités de la structure spécifique dans les trois versants de la forêt du Karthala. Il est apparu une asymétrie de la structure spécifique dans les versants. En effet, On a noté une forte densité au Sud, une diversité floristique plus élevée à l'Ouest alors que la surface terrière est plus élevée à l'Est. Le taux d'endémisme varie aussi. Il est plus élevé à l'Ouest et plus faible à l'Est. $W$. comorensis qui présente une population importante et stable dans les trois versants, serait l'espèce la plus adaptée aux différents topos séquences. Il a été noté que la régénération est faible chez les espèces importantes du milieu. Bien qu'il apparaisse clair que l'exposition au vent influe sur le patron habitat de la forêt du Karthala et donc sur la structure des peuplements végétaux, il serait intéressant de poursuivre les études pour établir la relation entre la variabilité des types de sol, des usages des espèces mais aussi des stratégies ( $\mathrm{R}$ ou $\mathrm{K}$ ) développées par les espèces au niveau de chaque versant. 


\section{REFERENCES}

Adjanohoune J, Ake Asssi L, Ahmed A, Eyme J, Guinko S, Kayonga A, Keita A, Lebras M. 1982. Contribution aux Etudes Ethnobotaniques et Floristiques aux Comores. ACCT : Paris; 216 p.

Akpo LE, Gaston A, Grouzis M. 1995. Structure spécifique d'une végétation sahélienne. Cas de wiidu thiengoli (Ferlo, Sénégal). Bul. Mus. Hist. Nat. Apris, 1-2: 39-52.

Boullet V. 2007. Habitat flore et végétation : diversité et originalité. Conservatoire botanique national des Mascareignes, $53 \mathrm{p}$.

Charahabil MM, Lô M, Bassène E, Akpo LE. 2008. Caractéristiques de la flore et de la végétation ligneuses des forêts communautaires de la zone soudanosahélienne au Sénégal. Journal des Sciences et Technologies, 6(2): 72-78.

Charahabil MM, Yahaya I, Andilyat A, Labat JN, Akpo LE. 2010. Typologie de la végétation ligneuse de la forêt $\mathrm{du}$ karthala, Archipel des Comores. XIXe Congrès de l'AETFAT, Madagascar, 2630 avril 2010. p 111.

Devineau JL, Lecordier C, Vuattoux R. 1984. Évolution de la diversité spécifique du peuplement ligneux dans une succession préforestière de colonisation d'une savane protégée des feux (Lamto, Côte d'Ivoire). Candollea, 39: 103- 134.

Diatta M, Grousis M, Faye E. 1998. Typologie de la végétation ligneuse en zone soudanienne. Bois et Forêts des Tropiques, 257(3) : 23-32.

Diouf M, Do Frederic, Akpo LE, Goudiaby V, Rocheteau A. 2004. Variation spatial de la structure spécifique d'un peuplement ligneux en milieux sahélien (Ferlo, NordSénégal) Ann. Bot. Afr. Ouest, 03: 75-86.

Ducatillon C, Loup C. 1985. Pratiques Paysannes des Comores. L'Arbre dans le
Paysage Agricole. IRAM-GRET: Paris, France; $98 \mathrm{p}$.

Holl K. 2002. Tropical moist forest. In Handbook of Ecological RestorationRestoration in Practice (vol. 2). Cambridge University Press: Cambridge 539- 558.

Keith P, Abdou A, Labat JN. 2006. Inventaire faunistique des rivières des Comores et inventaires botaniques. Muséum National d'histoire naturelle de Paris.

Labat J-N, Pascal O. 1999. Two new species of Cynometra (Fabaceae: Caesalpinioideae) from Mayotte in the Comoro Archipelago. Kew Bulletin, 54(1): 163-169.

Labat J-N, Du Puy DJ. 1997. New species and varieties in Sesbania (LeguminosaePapilionoideae-Robinieae) from Madagascar and the Comoro Islands. Adansonia, 19(1): 93-99.

Labat J-N, Pignal M, Rouhan Germinal, Yahaya I, Andy A, Loup C, Bidault E. 2010. Le patrimoine naturel caché des Comores : passé, présent et avenir de la diversité des plantes vasculaires. Scripta Bot Belg., 46.

Pascal O, Labat JN, Pignal M. 2001. Diversité, affinités phytogéographiques et origine présumée de la flore de Mayotte (Archipel des Comores). Syst. Geogr. Pl., 71: 1101-1123.

Pascal O. 1997. La Végétation Naturelle à Mayotte, Etudes Quantitatives et Qualitatives. DAF, SEF: Mayotte; 90 p.

Pascal O. 2002. Plantes et forêts de Mayotte. Patrimoines Naturels 53, MNHN, IEGB, $108 \mathrm{p}$.

Rajemison AH. 2010. Typologie de la végétation rémanente en vue d'une restauration écologique. Cas du site d'Ankafobe - Tampoketsa d'Ankazobe Hautes terres centrales de Madagascar. Mémoire de DEA, Ecole supérieur des 
Sciences agronomiques d'Antananarivo, 83p.

Ramade F. 1984. Eléments d'Ecologie. Ecologie Fondamentale. Me Graw-Hill: Paris.

Sarrasin B. 2006. Économie politique du développement minier à Madagascar : l'analyse du projet QMM à Tolagnaro. VertigO - la Revue Electronique en Sciences de l'Environnement, 7(2), en ligne.

Trochain V. 1996. Hétérogénéité spatiale des structures en forêt naturelle en basse altitude à Sumatra. Thèse de doctorat $3^{\text {ème }}$ cycle Université de Toulouse III, 268p.
Vos P. 2004. Etudes des plantes ligneuses envahissantes de l'archipel des Comores (Union des Comores et Mayotte). Note thématique sur la santé des forêts et la biosécurité. FAO, 42p.

Zainaba A. 2009. Contribution à l'étude de la pharmacopée traditionnelle des Comores : Enquête ethnobotanique dans la région de Hamahame. Thèse de Doctorat en Pharmacie, 128p. 